\title{
Mortality Risk within 14 Days after Coronavirus Disease 2019 Diagnosis in Dementia Patients: A Nationwide Analysis
}

\author{
Yi-Jun Kim ${ }^{\mathrm{a}}$ Yongho Jee ${ }^{\mathrm{b}}$ Sholhui Park ${ }^{\mathrm{c}}$ Eun-Hee Ha ${ }^{\mathrm{d}} \mathrm{e}$ Inho Jo ${ }^{\mathrm{e}, \mathrm{f}}$ \\ Hyang Woon Lee ${ }^{e, g}$ Myung Seon Song ${ }^{h}$
}

\begin{abstract}
anstitute of Convergence Medicine, Ewha Womans University Mokdong Hospital, Seoul, Republic of Korea;
${ }^{b}$ Advanced Biomedical Research Institute, Ewha Womans University Seoul Hospital, Seoul, Republic of Korea; 'Department of Laboratory Medicine, Ewha Womans University Seoul Hospital, Seoul, Republic of Korea; dDepartment of Occupational and Environmental Medicine, Ewha Womans University College of Medicine, Seoul, Republic of Korea; 'System Health Science \& Engineering, Ewha Womans University, Seoul, Republic of Korea; fDepartment of Molecular Medicine, Ewha Womans University College of Medicine, Seoul, Republic of Korea; gDepartments of Neurology, Medical Science, and Computational Medicine, Ewha Womans University College of

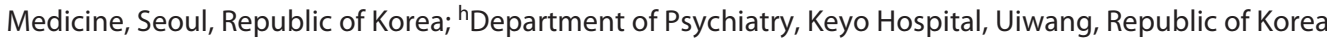

\section{Keywords}

Dementia · COVID-19 · Early death · Propensity score matching $\cdot$ General logistic regression

\begin{abstract}
Introduction: The study evaluated the increased mortality risk within 14 days of coronavirus disease 2019 (COVID-19) diagnosis in dementia patients. Methods: This retrospective study was conducted from February to April 2020 using the COVID-19 patients' database from the Korea Disease Control and Prevention Agency. The risk factors for early death within 14 days were determined using generalized logistic regression performed in a stepwise manner. Dementia patients diagnosed with COVID-19 were used for the study. The propensity score-matched cohort was included as controls. The differences in mortality within 14 days after COVID-19 diagnosis between the dementia patients and controls were evaluated. Results: We enrolled 5,349 COVID-19 patients from the database; 224 had dementia as comorbidity. The mortality rate within 14 days after COVID-19 diagnosis in dementia patients and the controls was $23.7 \%$ versus $1.7 \%$, re-
\end{abstract}

karger@karger.com

(c) 2021 S. Karger AG, Basel

www.karger.com/dem

Karger" spectively, before propensity score matching (PSM) $(p<$ $0.001)$, and $23.7 \%$ versus $9.2 \%$ after PSM $(p<0.001)$. The hazard ratio (HR) for mortality within 14 days in COVID-19 patients with dementia was significant even after PSM (HR $5.104,95 \%$ confidence interval $2.889-5.673, p<0.001)$. The survival curve of dementia patients was steeply inclined within 14 days after COVID-19 diagnosis, resulting in 70.7\% of all deaths in dementia patients. Conclusions: COVID-19 patients with dementia had a higher risk of early death within 14 days. Thus, prompt intervention is necessary for dementia patients after COVID-19 diagnosis.

(c) 2021 S. Karger AG, Basel

\section{Introduction}

The coronavirus disease 2019 (COVID-19) caused by severe acute respiratory syndrome coronavirus 2 infection is hitting the world. In August 2021, 204 million cases and 4.31 million deaths were reported worldwide [1].

Yi-Jun Kim and Yongho Jee contributed equally to this work.
Correspondence to:

Yi-Jun Kim, kimyj.ro@gmail.com 
Since no herd immunity of this pandemic exists, COVID-19 rapid spread before the lockdown in many countries of the world was significantly higher than influenza, or even measles spread [2]. On December 11, 2020, the US Food and Drug Administration issued the first authorization for emergency vaccination to prevent COVID-19 $[3,4]$. Although vaccine distribution has begun, it will take years to get enough vaccines worldwide $[1,5]$. Therefore, efficient patient treatment will continually be necessary amidst limited medical resources.

To date, several studies have analyzed poor COVID-19 prognostic risk factors [6, 7]. However, survivor and nonsurvivor characteristics are most merely analyzed and compared $[6,7]$. Early intensive care intervention is possible for more emergency patients with death possibility within a few weeks (within 14 days) after diagnoses. To date, no papers have reported on mortality within a specific period after COVID-19 diagnosis.

Individuals with $A P O E \varepsilon 4 / \varepsilon 4$ homozygotes were more likely to test positive for COVID-19, with higher mortality than other genotypes [8]. This variant is a common risk factor for Alzheimer's disease, the most common form of dementia $[9,10]$. This suggests higher COVID-19 susceptibility, and fatality in dementia patients is not only social but also genetic. Therefore, dementia patients' mortality pattern in COVID-19 may differ from other deaths. Several studies have shown dementia as a poor COVID-19 risk factor. However, none has shown dementia's association with early death within a few weeks of COVID-19 diagnosis [11, 12].

The Korea Disease Control and Prevention Agency (KDCA) released COVID-19 patients' data from February to April 2020 across the country for research purposes. Thus, we characterized the death cases within 14 and 30 days of COVID-19 diagnosis to determine early mortality risk factors among dementia patients. The aim of this study was to elucidate risk factors for early death after COVID-19 infection and to identify dementia as one of the independent risk factors for early death after COVID-19 infection.

\section{Materials and Methods}

\section{Study Design and Setting}

Patients

The KDCA provided COVID-19 patients' data from February to April 30, 2020. The anonymized data were available to authorized researchers using a virtual server. All patients were hospitalized, treated, quarantined, and finally discharged as dead or alive. Patients diagnosed with COVID-19 after death and patients with missing data were excluded from the study. Demographic infor- mation, initial examination findings, clinical results on admission, comorbid diseases, clinical severity, and general blood test results were provided.

\section{Study Variable Description}

The study variables include demographics characteristics, such as age, sex, and body mass index (BMI), and patients' vital signs, including systolic blood pressure, diastolic blood pressure, heart rate, and temperature. COVID-19 symptoms (fever, cough, sputum, sore throat, runny nose, myalgia, fatigue, shortness of breath, headache, change of consciousness, vomiting/nausea, and diarrhea) and comorbid diseases (diabetes mellitus, hypertension, heart failure, other chronic heart diseases, asthma, chronic obstructive pulmonary disease, chronic kidney disease, cancer, chronic liver disease, rheumatoid disease/autoimmune disease, and dementia) were included in the study. The use of the intensive care unit during hospitalization and blood test results (hemoglobin, hematocrit, lymphocyte, platelets, and white blood cell) were analyzed.

\section{Statistical Analysis}

Pearson's $\chi^{2}$ test was used to compare the differences between categorical variables and prognostic outcomes between dementia patients and the control. Blood test results were classified according to the normal and abnormal ranges of the test [13]. Generalized logistic regression was performed in a stepwise manner after incorporating all variables to determine the risk factors for early mortality within 30 days. Variables incorporated were demographics, vital sign, COVID-19 symptoms, comorbid diseases, and blood test results. Subgroups with normal or normal-low variable values were selected as reference groups. The same analysis was performed for early death within 14 days.

To further analyze the difference in early mortality according to the prevalence of dementia, propensity score matching (PSM) was performed. A 2:1 control:dementia ratio using "optimal" matching was conducted. We analyzed the differences between the matched control and dementia patients' mortality at 14 and 30 days after COVID-19 diagnosis. A well-balanced match was defined as a standardized mean difference of $<0.25$ and a statistically insignificant $p$ value of the $\chi^{2}$ test between the matched control and the dementia patients. Survival estimate was performed using the Kaplan-Meier estimate and compared by a log-rank test. Twotailed $p$ value $<0.05$ was considered statistically significant. All analyses were performed using $\mathrm{R}$ programming software (ver. 4.0.2). The "MatchIt" R package was used for PSM.

\section{Results}

\section{Patient Characteristics}

A total of 5,628 COVID-19 patients were diagnosed. Of these, 7 patients with COVID-19 diagnosis after death were excluded; 5,349 patients remained after excluding patients aged $<20$ years old. Patient characteristics are summarized in Table 1. Patients over 70 years of age were $16.2 \%$, and females were $59.4 \%$. The most common symptoms at the time of COVID-19 diagnosis were fever 
Table 1. Patient characteristics at the time of COVID-19 diagnosis

\begin{tabular}{|c|c|c|c|}
\hline Patient characteristics & Values & Patient characteristics & Values \\
\hline Demographics and vital sign, $n(\%)$ & & Shortness of breath & \\
\hline Age, years & & No & $4,689(87.7)$ \\
\hline$<50$ & $2,425(45.3)$ & Yes & $656(12.3)$ \\
\hline$\geq 50$ and $<70$ & $2,059(38.5)$ & Unknown & $4(0.1)$ \\
\hline$\geq 70$ & $865(16.2)$ & Headache & \\
\hline Sex & & No & $4,399(82.2)$ \\
\hline Male & $2,172(40.6)$ & Yes & $946(17.7)$ \\
\hline Female & $3,177(59.4)$ & Unknown & $4(0.1)$ \\
\hline BMI & & Change of consciousness & \\
\hline $\operatorname{Low}(<18.5)$ & $193(3.6)$ & No & $5,313(99.3)$ \\
\hline Normal low ( $\geq 18.5$ and $<23.0$ ) & $1,762(32.9)$ & Yes & $32(0.6)$ \\
\hline Normal high $(\geq 23.0$ and $<25.0$ ) & $1,012(18.9)$ & Unknown & $4(0.1)$ \\
\hline High $(\geq 25.0$ and $<30.0)$ & $1,021(19.1)$ & Vomiting/nausea & \\
\hline Higher $(\geq 30.0)$ & $194(3.6)$ & No & $5,106(95.5)$ \\
\hline Unknown & $1,167(21.8)$ & Yes & $239(4.5)$ \\
\hline Systolic blood pressure, $\mathrm{mm} \mathrm{Hg}$ & & Unknown & $4(0.1)$ \\
\hline Low $(<120)$ & $1,220(22.8)$ & Diarrhea & \\
\hline Normal low ( $\geq 120$ and $<130$ ) & $1,090(20.4)$ & No & $4,838(90.4)$ \\
\hline Normal high ( $\geq 130$ and $<140$ ) & $1,048(19.6)$ & Yes & $507(9.5)$ \\
\hline High $(\geq 140$ and $<160)$ & $1,385(25.9)$ & Unknown & $4(0.1)$ \\
\hline Higher $(\geq 160)$ & $507(9.5)$ & Comorbidity at the time of COVID-19 diagnosis, $n(\%)$ & \\
\hline Unknown & $99(1.9)$ & Diabetes mellitus & \\
\hline Diastolic blood pressure, $\mathrm{mm} \mathrm{Hg}$ & & No & $4,660(87.1)$ \\
\hline Low $(<80)$ & $1,990(37.2)$ & Yes & $686(12.8)$ \\
\hline Normal low ( $\geq 80$ and $<90)$ & $1,735(32.4)$ & Unknown & $3(0.1)$ \\
\hline Normal high ( $\geq 90$ and $<100$ ) & $1,032(19.3)$ & Hypertension & \\
\hline High $(\geq 100)$ & $493(9.2)$ & No & $4,148(77.5)$ \\
\hline Unknown & 99 (1.9) & Yes & $1,198(22.4)$ \\
\hline Heart rate, beats per min & & Unknown & $3(0.1)$ \\
\hline Low $(<60)$ & $107(2.0)$ & Heart failure & \\
\hline Normal low ( $\geq 60$ and $<80$ ) & $1,811(33.9)$ & No & $5,288(98.9)$ \\
\hline Normal high ( $\geq 80$ and $<100$ ) & $2,439(45.6)$ & Yes & $58(1.1)$ \\
\hline High $(\geq 100)$ & $882(16.5)$ & Unknown & $3(0.1)$ \\
\hline Unknown & $110(2.1)$ & Other chronic heart disease & \\
\hline Temperature, ${ }^{\circ} \mathrm{C}$ & & No & $5,151(96.3)$ \\
\hline $\operatorname{Low}(<36.0)$ & $22(0.4)$ & Yes & $179(3.3)$ \\
\hline Normal low ( $\geq 36.0$ and $<37.0$ ) & $2,883(53.9)$ & Unknown & $19(0.4)$ \\
\hline Normal high $(\geq 37.0$ and $<38.0$ ) & $2,131(39.8)$ & Asthma & \\
\hline High $(\geq 38.0)$ & $277(5.2)$ & No & $5,220(97.6)$ \\
\hline Unknown & $36(0.7)$ & Yes & $126(2.4)$ \\
\hline Symptoms at the time of COVID-19 diagnosis, $n$ (\%) & & Unknown & $3(0.1)$ \\
\hline Fever & & Chronic obstructive pulmonary disease & \\
\hline No & $4,098(76.6)$ & No & $5,308(99.2)$ \\
\hline Yes & $1,247(23.3)$ & Yes & $38(0.7)$ \\
\hline Unknown & $4(0.1)$ & Unknown & $3(0.1)$ \\
\hline Cough & & Chronic kidney disease & \\
\hline No & $3,099(57.9)$ & No & $5,291(98.9)$ \\
\hline Yes & $2,246(42.0)$ & Yes & $55(1.0)$ \\
\hline Unknown & $4(0.1)$ & Unknown & $3(0.1)$ \\
\hline Sputum & & Cancer & \\
\hline No & $3,783(70.7)$ & No & $5,202(97.3)$ \\
\hline Yes & $1,562(29.2)$ & Yes & $143(2.7)$ \\
\hline Unknown & $4(0.1)$ & Unknown & $4(0.1)$ \\
\hline Sore throat & & Chronic liver disease & \\
\hline No & $4,497(84.1)$ & No & 4,952 (92.6) \\
\hline Yes & $848(15.9)$ & Yes & $82(1.5)$ \\
\hline Unknown & $4(0.1)$ & Unknown & $315(5.9)$ \\
\hline Runny nose & & Rheumatoid disease/autoimmune disease & \\
\hline No & $4,769(89.2)$ & No & $4,990(93.3)$ \\
\hline Yes & $576(10.8)$ & Yes & $38(0.7)$ \\
\hline Unknown & $4(0.1)$ & Unknown & $321(6.0)$ \\
\hline Myalgia & & Dementia & \\
\hline No & $4,433(82.9)$ & No & 4,807 (89.9) \\
\hline Yes & $912(17.0)$ & Yes & $224(4.2)$ \\
\hline Unknown & $4(0.1)$ & Unknown & $318(5.9)$ \\
\hline \multicolumn{4}{|l|}{ Fatigue } \\
\hline No & $5,115(95.6)$ & & \\
\hline Yes & $230(4.3)$ & & \\
\hline Unknown & $4(0.1)$ & & \\
\hline
\end{tabular}


Table 1 (continued)

\begin{tabular}{|c|c|}
\hline Patient characteristics & Values \\
\hline \multicolumn{2}{|l|}{ Blood test, $n(\%)$} \\
\hline \multicolumn{2}{|l|}{ Hemoglobin, $\mathrm{g} / \mathrm{dL}$} \\
\hline Lower $($ men $<12.3$; women $<10.8$ ) & $197(3.7)$ \\
\hline Low (men $\geq 12.3-14.0 ;$ women $\geq 10.8$ and $<12.3$ ) & $807(15.1)$ \\
\hline $\begin{array}{l}\text { Normal low (men } \geq 14.0 \text { and }<15.7 \text {; women } \geq 12.3 \text { and } \\
<13.8 \text { ) }\end{array}$ & $2,073(38.8)$ \\
\hline $\begin{array}{l}\text { Normal high (men } \geq 15.7 \text { and }<17.5 \text {; women } \geq 13.8 \text { and } \\
<15.3 \text { ) }\end{array}$ & $760(14.2)$ \\
\hline High (men $\geq 17.5 ;$ women $\geq 15.3$ ) & $58(1.1)$ \\
\hline Unknown & $1,454(27.2)$ \\
\hline \multicolumn{2}{|l|}{ Hematocrit, \% } \\
\hline Low (men <41.5; women <35.9) & $1,332(24.9)$ \\
\hline $\begin{array}{l}\text { Normal low (men } \geq 41.5 \text { and }<45.9 \text {; women } \geq 35.9 \text { and } \\
<40.2 \text { ) }\end{array}$ & $1,613(30.2)$ \\
\hline $\begin{array}{l}\text { Normal high (men } \geq 45.9 \text { and }<50.4 \text {; women } \geq 40.2 \text { and } \\
<44.6 \text { ) }\end{array}$ & $839(15.7)$ \\
\hline High (men $\geq 50.4$; women $\geq 44.6$ ) & $105(2.0)$ \\
\hline Unknown & $1,460(27.3)$ \\
\hline \multicolumn{2}{|l|}{ Lymphocyte, \% } \\
\hline Low $(<20)$ & $868(16.2)$ \\
\hline Normal low ( $\geq 20$ and $<32$ ) & $1,545(28.9)$ \\
\hline Normal high ( $\geq 32$ and $<44$ ) & $1,147(21.4)$ \\
\hline High $(\geq 44)$ & $311(5.8)$ \\
\hline Unknown & $1,478(27.6)$ \\
\hline \multicolumn{2}{|l|}{ Platelets, $\mu \mathrm{L}$} \\
\hline Low $(<150 K)$ & $486(9.1)$ \\
\hline Normal low ( $\geq 150 \mathrm{~K}$ and $<300 \mathrm{~K})$ & $2,678(50.1)$ \\
\hline Normal high ( $\geq 300 \mathrm{~K}$ and $<450 \mathrm{~K})$ & $669(12.5)$ \\
\hline High $(\geq 450 \mathrm{~K})$ & $63(1.2)$ \\
\hline Unknown & $1,453(27.2)$ \\
\hline \multicolumn{2}{|l|}{ White blood cell, $\mu \mathrm{L}$} \\
\hline Low $(<4,500)$ & $1,017(19.0)$ \\
\hline Normal $(\geq 4,500$ and $<11,000)$ & $2,727(51.0)$ \\
\hline High $(\geq 11,000)$ & $152(2.8)$ \\
\hline Unknown & $1,453(27.2)$ \\
\hline \multicolumn{2}{|l|}{ Outcomes, $n(\%)$} \\
\hline \multicolumn{2}{|l|}{ Using the intensive care unit during hospitalization } \\
\hline No & $5,135(96.0)$ \\
\hline Yes & $187(3.5)$ \\
\hline Unknown & $27(0.5)$ \\
\hline \multicolumn{2}{|l|}{ Death, $N$} \\
\hline No & $5,115(95.6)$ \\
\hline Yes & $234(4.4)$ \\
\hline \multicolumn{2}{|l|}{ Death within 30 days } \\
\hline No & $5,152(96.3)$ \\
\hline Yes & $197(3.7)$ \\
\hline \multicolumn{2}{|l|}{ Death within 14 days } \\
\hline No & $5,208(97.4)$ \\
\hline Yes & $141(2.6)$ \\
\hline
\end{tabular}

COVID-19, coronavirus disease 2019; BMI, body mass index.

(23.3\%), cough (42.0\%), and sputum (29.2\%), while some presented with shortness of breath (12.3\%) and change of consciousness $(0.6 \%)$. Among the patients, $3.5 \%$ received intensive care during the hospitalization. Death occurred in 234 (4.4\%) of 5,349 patients, 197 (3.7\%) within 30 days, and $141(2.6 \%)$ within 14 days.

At COVID-19 diagnosis, 224 patients (4.2\%) had dementia (Table 2). Most dementia patients were older than 70 years $(85.7 \%)$, compared to $86.9 \%$ of the control group younger than 70 years. Dementia patients were more likely women (59.0\% vs. $68.3 \%)$. The underweight population in the dementia group was 2.5 times that of the control group ( $3.4 \%$ vs. $8.9 \%)$, and the proportions of low diastolic blood pressure and low heart rate were higher than those of the control group (diastolic blood pressure $<80 \mathrm{~mm} \mathrm{Hg}, 36.8 \%$ vs. $46.9 \%$; heart rate $<60$ beats per min, $1.7 \%$ vs. $8.0 \%$, respectively). The rates of underlying diseases including diabetes, high blood pressure, heart disease, lung disease, and kidney disease were higher than those of the control group. In contrast, dementia patients had significantly fewer complaints of symptoms such as cough, sputum, sore throat, runny nose, myalgia, and headache than the control group. The rate of loss of consciousness symptoms was higher in dementia patients $(0.5 \%$ vs. $3.6 \%)$. In blood test, dementia patients had a higher percentage of below normal levels of hemoglobin, hematocrit, lymphocyte, platelet, and white blood cells than the control group.

\section{Dementia Was a Significant Mortality Risk Factor}

\section{within 30 and 14 Days after COVID-19 Diagnosis}

General logistic regression analysis for death within 30 days after COVID-19 diagnosis shows that dementia was a significant risk factor (hazard ratio [HR] 4.543, 95\% confidence interval [CI] 2.788-5.031, $p<0.001$ ) (Table 3). Old age, low BMI, low or high body temperature, shortness of breath, change of consciousness, diabetes mellitus, cancer, low hemoglobin, low lymphocyte, low platelets, and high white blood cell were also significant risk factors for mortality within 30 days. On the other hand, female sex, sore throat, and headache were favorable prognostic factors.

Factors related to death within 14 days were also confirmed by the same analysis, and dementia was still a significant risk factor (HR 5.175, 95\% CI 3.073-5.696, $p<$ 0.001) (Table 4). Low BMI and cancer, which were significant risk factors for death within 30 days, were excluded from significant risk factors for mortality within 14 days. Old age, low or high body temperature, shortness of breath, change of consciousness, diabetes mellitus, low hemoglobin, low lymphocyte, low platelet, and high white blood cell were identified as significant risk factors for death within 14 days, while headache was a significantly good prognostic factor.

\section{Early Mortality Between Dementia Patients and Matched Control}

Of the 224 dementia patients, 75 (33.5\%) deaths occurred (Table 2). This accounts for $32.1 \%$ of 234 deaths. 
Table 2. Patient characteristics before and after PSM according to dementia in COVID-19

\begin{tabular}{|c|c|c|c|c|c|c|c|c|}
\hline \multirow[t]{2}{*}{ Characteristics } & \multicolumn{4}{|l|}{ Before PSM } & \multicolumn{4}{|l|}{ After PSM } \\
\hline & $\begin{array}{l}\text { control } \\
(N=5,125)\end{array}$ & $\begin{array}{l}\text { dementia } \\
(N=224)\end{array}$ & $p$ value & SMD & $\begin{array}{l}\text { control } \\
(N=448)\end{array}$ & $\begin{array}{l}\text { dementia } \\
(N=224)\end{array}$ & $p$ value & SMD \\
\hline \multicolumn{9}{|l|}{ Demographics and vital sign, $n(\%)$} \\
\hline \multicolumn{9}{|l|}{ Age, years } \\
\hline$<50$ & $2,424(47.3)$ & $1(0.4)$ & \multirow{3}{*}{$<0.001$} & 23.076 & $2(0.4)$ & $1(0.4)$ & \multirow{3}{*}{0.623} & $<0.001$ \\
\hline$\geq 50$ and $<70$ & $2,028(39.6)$ & $31(13.8)$ & & 2.113 & $75(16.7)$ & $31(13.8)$ & & 0.063 \\
\hline$\geq 70$ & $673(13.1)$ & $192(85.7)$ & & 1.746 & $371(82.8)$ & $192(85.7)$ & & 0.061 \\
\hline \multicolumn{9}{|l|}{ Sex } \\
\hline Male & $2,101(41.0)$ & $71(31.7)$ & \multirow[t]{2}{*}{0.007} & 0.523 & $171(38.2)$ & $71(31.7)$ & \multirow[t]{2}{*}{0.118} & 0.142 \\
\hline Female & $3,024(59.0)$ & $153(68.3)$ & & 0.434 & $277(61.8)$ & $153(68.3)$ & & 0.135 \\
\hline $\mathrm{BMI}$ & & & & & & & & \\
\hline Low $(<18.5)$ & $173(3.4)$ & $20(8.9)$ & & 0.182 & $34(7.6)$ & $20(8.9)$ & & 0.027 \\
\hline Normal low ( $\geq 18.5$ and $<23.0$ ) & $1,697(33.1)$ & $65(29.0)$ & & 0.217 & $138(30.8)$ & $65(29.0)$ & & 0.038 \\
\hline Normal high $(\geq 23.0$ and $<25.0)$ & $992(19.4)$ & $20(8.9)$ & $<0.001$ & 0.749 & $54(12.1)$ & $20(8.9)$ & 0.345 & 0.070 \\
\hline High $(\geq 25.0$ and $<30.0)$ & $1,005(19.6)$ & $16(7.1)$ & & 1.003 & $46(10.3)$ & $16(7.1)$ & & 0.071 \\
\hline Higher $(\geq 30.0)$ & $194(3.8)$ & $0(0.0)$ & & NA & $1(0.2)$ & $0(0.0)$ & & NA \\
\hline Unknown & $1,064(20.8)$ & $103(46)$ & & 0.889 & $175(39.1)$ & $103(46.0)$ & & 0.143 \\
\hline Systolic blood pressure, $\mathrm{mm} \mathrm{Hg}$ & & & & & & & & \\
\hline Low $(<120)$ & $1,166(22.8)$ & $54(24.1)$ & & 0.066 & $84(18.8)$ & $54(24.1)$ & & 0.109 \\
\hline Normal low ( $\geq 120$ and $<130$ ) & $1,053(20.5)$ & $37(16.5)$ & & 0.222 & $75(16.7)$ & $37(16.5)$ & & 0.005 \\
\hline Normal high ( $\geq 130$ and $<140)$ & $1,013(19.8)$ & 35 (15.6) & 0.119 & 0.230 & $71(15.8)$ & $35(15.6)$ & 0.705 & 0.005 \\
\hline High $(\geq 140$ and $<160)$ & $1,320(25.8)$ & $65(29.0)$ & & 0.154 & $146(32.6)$ & $65(29.0)$ & & 0.077 \\
\hline Higher $(\geq 160)$ & $477(9.3)$ & 30 (13.4) & & 0.173 & $64(14.3)$ & $30(13.4)$ & & 0.019 \\
\hline Unknown & $96(1.9)$ & $3(1.3)$ & & 0.031 & $8(1.8)$ & $3(1.3)$ & & 0.010 \\
\hline Diastolic blood pressure, $\mathrm{mm} \mathrm{Hg}$ & & & & & & & & \\
\hline Low $(<80)$ & $1,885(36.8)$ & $105(46.9)$ & & 0.451 & $192(42.9)$ & $105(46.9)$ & & 0.084 \\
\hline Normal low ( $\geq 80$ and $<90$ ) & $1,667(32.5)$ & $68(30.4)$ & & 0.112 & $138(30.8)$ & $68(30.4)$ & & 0.009 \\
\hline Normal high ( $\geq 90$ and $<100$ ) & $997(19.5)$ & $35(15.6)$ & 0.025 & 0.211 & $81(18.1)$ & $35(15.6)$ & 0.850 & 0.053 \\
\hline High $(\geq 100)$ & $480(9.4)$ & $13(5.8)$ & & 0.222 & $29(6.5)$ & $13(5.8)$ & & 0.014 \\
\hline Unknown & $96(1.9)$ & $3(1.3)$ & & 0.031 & $8(1.8)$ & $3(1.3)$ & & 0.010 \\
\hline Heart rate, beats per min & & & & & & & & \\
\hline Low $(<60)$ & $89(1.7)$ & $18(8.0)$ & & 0.168 & $20(4.5)$ & $18(8.0)$ & & 0.071 \\
\hline Normal low ( $\geq 60$ and $<80$ ) & $1,720(33.6)$ & $91(40.6)$ & & 0.323 & $164(36.6)$ & $91(40.6)$ & & 0.084 \\
\hline Normal high $(\geq 80$ and $<100)$ & $2,351(45.9)$ & $88(39.3)$ & $<0.001$ & 0.353 & $192(42.9)$ & $88(39.3)$ & 0.189 & 0.077 \\
\hline High $(\geq 100)$ & $858(16.7)$ & $24(10.7)$ & & 0.370 & $65(14.5)$ & $24(10.7)$ & & 0.085 \\
\hline Unknown & $107(2.1)$ & $3(1.3)$ & & 0.046 & $7(1.6)$ & $3(1.3)$ & & 0.005 \\
\hline Temperature, ${ }^{\circ} \mathrm{C}$ & & & & & & & & \\
\hline Low $(<36.0)$ & $20(0.4)$ & $2(0.9)$ & & 0.017 & $5(1.1)$ & $2(0.9)$ & & 0.005 \\
\hline Normal low ( $\geq 36.0$ and $<37.0)$ & $2,733(53.3)$ & $150(67.0)$ & & 0.614 & $290(64.7)$ & $150(67.0)$ & & 0.047 \\
\hline Normal high ( $\geq 37.0$ and $<38.0$ ) & $2,073(40.4)$ & $58(25.9)$ & $<0.001$ & 0.894 & $120(26.8)$ & $58(25.9)$ & 0.968 & 0.019 \\
\hline High $(\geq 38.0)$ & $266(5.2)$ & $11(4.9)$ & & 0.014 & $27(6.0)$ & $11(4.9)$ & & 0.024 \\
\hline Unknown & $33(0.6)$ & $3(1.3)$ & & 0.025 & $6(1.3)$ & $3(1.3)$ & & $<0.001$ \\
\hline Symptoms at the time of COVID-19 diag & & & & & & & & \\
\hline Fever & & & & & & & & \\
\hline No/unknown & $3,923(76.5)$ & $179(79.9)$ & 0.278 & 0.165 & $363(81)$ & 179 (79.9) & 0.809 & 0.024 \\
\hline Yes & $1,202(23.5)$ & $45(20.1)$ & & 0.180 & $85(19)$ & $45(20.1)$ & & 0.023 \\
\hline Cough & & & & & & & & \\
\hline No/unknown & $2,920(57.0)$ & $183(81.7)$ & $<0.001$ & 1.049 & $344(76.8)$ & $183(81.7)$ & 0.174 & 0.103 \\
\hline Yes & $2,205(43.0)$ & $41(18.3)$ & & 1.846 & $104(23.2)$ & $41(18.3)$ & & 0.109 \\
\hline Sputum & & & & & & & & \\
\hline No/unknown & $3,591(70.1)$ & $196(87.5)$ & $<0.001$ & 0.787 & $374(83.5)$ & $196(87.5)$ & 0.210 & 0.085 \\
\hline Yes & $1,534(29.9)$ & $28(12.5)$ & & 1.312 & $74(16.5)$ & $28(12.5)$ & & 0.090 \\
\hline Sore throat & & & & & & & & \\
\hline No/unknown & $4,281(83.5)$ & $220(98.2)$ & $<0.001$ & 0.681 & $434(96.9)$ & $220(98.2)$ & 0.447 & 0.028 \\
\hline Yes & $844(16.5)$ & $4(1.8)$ & & 2.142 & $14(3.1)$ & $4(1.8)$ & & 0.031 \\
\hline Runny nose & & & & & & & & \\
\hline No/unknown & $4,551(88.8)$ & $222(99.1)$ & $<0.001$ & 0.489 & $439(98.0)$ & $222(99.1)$ & 0.452 & 0.024 \\
\hline Yes & $574(11.2)$ & $2(0.9)$ & & 1.751 & $9(2.0)$ & $2(0.9)$ & & 0.028 \\
\hline Myalgia & & & & & & & & \\
\hline No/unknown & $4,219(82.3)$ & $218(97.3)$ & $<0.001$ & 0.694 & $374(83.5)$ & $196(87.5)$ & 0.814 & 0.085 \\
\hline Yes & $906(17.7)$ & $6(2.7)$ & & 1.854 & $74(16.5)$ & $28(12.5)$ & & 0.087 \\
\hline Fatigue & & & & & & & & \\
\hline No/unknown & $4,906(95.7)$ & $213(95.1)$ & 0.770 & 0.032 & $425(94.9)$ & $213(95.1)$ & 1.000 & 0.005 \\
\hline Yes & $219(4.3)$ & $11(4.9)$ & & 0.030 & $23(5.1)$ & $11(4.9)$ & & 0.005 \\
\hline Shortness of breath & & & & & & & & \\
\hline No/unknown & $4,506(87.9)$ & $187(83.5)$ & 0.060 & 0.227 & $362(80.8)$ & $187(83.5)$ & 0.459 & 0.056 \\
\hline Yes & $619(12.1)$ & $37(16.5)$ & & 0.192 & $86(19.2)$ & $37(16.5)$ & & 0.058 \\
\hline Headache & & & & & & & & \\
\hline No/unknown & $4,186(81.7)$ & 217 (96.9) & $<0.001$ & 0.702 & $429(95.8)$ & 217 (96.9) & 0.621 & 0.024 \\
\hline Yes & 939 (18.3) & $7(3.1)$ & & 1.772 & $19(4.2)$ & $7(3.1)$ & & 0.025 \\
\hline
\end{tabular}


Table 2 (continued)

\begin{tabular}{|c|c|c|c|c|c|c|c|c|}
\hline \multirow[t]{2}{*}{ Characteristics } & \multicolumn{4}{|l|}{ Before PSM } & \multicolumn{4}{|l|}{ After PSM } \\
\hline & $\begin{array}{l}\text { control } \\
(N=5,125)\end{array}$ & $\begin{array}{l}\text { dementia } \\
(N=224)\end{array}$ & $p$ value & SMD & $\begin{array}{l}\text { control } \\
(N=448)\end{array}$ & $\begin{array}{l}\text { dementia } \\
(N=224)\end{array}$ & $p$ value & SMD \\
\hline \multicolumn{9}{|l|}{ Change of consciousness } \\
\hline No/unknown & $5,101(99.5)$ & $216(96.4)$ & $<0.001$ & 0.157 & $436(97.3)$ & $216(96.4)$ & 0.688 & 0.019 \\
\hline Yes & $24(0.5)$ & $8(3.6)$ & & 0.071 & $12(2.7)$ & $8(3.6)$ & & 0.018 \\
\hline \multicolumn{9}{|l|}{ Vomiting/nausea } \\
\hline No/unknown & $4,893(95.5)$ & $217(96.9)$ & 0.407 & 0.070 & $424(94.6)$ & 217 (96.9) & 0.269 & 0.047 \\
\hline Yes & $232(4.5)$ & $7(3.1)$ & & 0.083 & $24(5.4)$ & $7(3.1)$ & & 0.053 \\
\hline \multicolumn{9}{|l|}{ Diarrhea } \\
\hline No/unknown & 4,631 (90.4) & $211(94.2)$ & 0.072 & 0.188 & $416(92.9)$ & $211(94.2)$ & 0.623 & 0.028 \\
\hline Yes & $494(9.6)$ & $13(5.8)$ & & 0.242 & $32(7.1)$ & $13(5.8)$ & & 0.029 \\
\hline \multicolumn{9}{|l|}{ Comorbidity at the time of COVID-19 diagnosis, $n(\%)$} \\
\hline \multicolumn{9}{|l|}{ Diabetes mellitus } \\
\hline No/unknown & $4,502(87.8)$ & $161(71.9)$ & $<0.001$ & 0.875 & $312(69.6)$ & $161(71.9)$ & 0.612 & 0.047 \\
\hline Yes & $623(12.2)$ & $63(28.1)$ & & 0.553 & $136(30.4)$ & $63(28.1)$ & & 0.048 \\
\hline \multicolumn{9}{|l|}{ Hypertension } \\
\hline No/unknown & $4,053(79.1)$ & $98(43.8)$ & $<0.001$ & 2.327 & $210(46.9)$ & $98(43.8)$ & 0.494 & 0.067 \\
\hline Yes & $1,072(20.9)$ & $126(56.3)$ & & 1.151 & $238(53.1)$ & $126(56.3)$ & & 0.066 \\
\hline \multicolumn{9}{|l|}{ Heart failure } \\
\hline No/unknown & $5,079(99.1)$ & $212(94.6)$ & $<0.001$ & 0.227 & $426(95.1)$ & $212(94.6)$ & 0.950 & 0.009 \\
\hline Yes & $46(0.9)$ & $12(5.4)$ & & 0.109 & $22(4.9)$ & $12(5.4)$ & & 0.009 \\
\hline \multicolumn{9}{|l|}{ Other chronic heart disease } \\
\hline No/unknown & 4,961 (96.8) & $209(93.3)$ & 0.008 & 0.178 & $416(92.9)$ & $209(93.3)$ & 0.957 & 0.009 \\
\hline Yes & $164(3.2)$ & $15(6.7)$ & & 0.126 & $32(7.1)$ & $15(6.7)$ & & 0.009 \\
\hline Asthma & & & & & & & & \\
\hline No/unknown & $5,007(97.7)$ & $216(96.4)$ & 0.317 & 0.064 & $434(96.9)$ & $216(96.4)$ & 0.939 & 0.009 \\
\hline Yes & $118(2.3)$ & $8(3.6)$ & & 0.052 & $14(3.1)$ & $8(3.6)$ & & 0.009 \\
\hline Chronic obstructive pulmonary disease & & & & & & & & \\
\hline No/unknown & $5,095(99.4)$ & $216(96.4)$ & $<0.001$ & 0.151 & $437(97.5)$ & $216(96.4)$ & 0.565 & 0.024 \\
\hline Yes & $30(0.6)$ & $8(3.6)$ & & 0.072 & $11(2.5)$ & $8(3.6)$ & & 0.022 \\
\hline Chronic kidney disease & & & & & & & & \\
\hline No/unknown & $5,080(99.1)$ & $214(95.5)$ & $<0.001$ & 0.182 & $432(96.4)$ & $214(95.5)$ & 0.724 & 0.019 \\
\hline Yes & $45(0.9)$ & $10(4.5)$ & & 0.092 & $16(3.6)$ & $10(4.5)$ & & 0.018 \\
\hline Cancer & & & & & & & & \\
\hline No/unknown & $4,987(97.3)$ & $219(97.8)$ & 0.839 & 0.027 & $438(97.8)$ & $219(97.8)$ & 1.000 & 0.000 \\
\hline Yes & $138(2.7)$ & $5(2.2)$ & & 0.025 & $10(2.2)$ & $5(2.2)$ & & 0.000 \\
\hline Chronic liver disease & & & & & & & & \\
\hline No/unknown & $5,049(98.5)$ & $218(97.3)$ & 0.251 & 0.060 & $438(97.8)$ & $218(97.3)$ & 0.929 & 0.009 \\
\hline Yes & $76(1.5)$ & $6(2.7)$ & & 0.046 & $10(2.2)$ & $6(2.7)$ & & 0.009 \\
\hline Rheumatoid disease/autoimmune disease & & & & & & & & \\
\hline No/unknown & $5,087(99.3)$ & $224(100)$ & 0.375 & 0.037 & $447(99.8)$ & $224(100)$ & 1.000 & 0.005 \\
\hline Yes & $38(0.7)$ & $0(0.0)$ & & NA & $1(0.2)$ & $0(0.0)$ & & NA \\
\hline Blood test, $n(\%)$ & & & & & & & & \\
\hline Hemoglobin, g/dL & & & & & & & & \\
\hline Lower (men <12.3; women <10.8) & $338(6.6)$ & 67 (29.9) & & 0.627 & $126(28.1)$ & $67(29.9)$ & & 0.037 \\
\hline Low (men $\geq 12.3-14.0$; women $\geq 10.8$ and $<12.3$ ) & $911(17.8)$ & $67(29.9)$ & & 0.480 & $130(29)$ & $67(29.9)$ & & 0.019 \\
\hline Normal low (men $\geq 14.0$ and $<15.7 ;$ women $\geq 12.3$ and $<13.8$ ) & $1,634(31.9)$ & $60(26.8)$ & $<0.001$ & 0.276 & $128(28.6)$ & $60(26.8)$ & 0.993 & 0.037 \\
\hline Normal high (men $\geq 15.7$ and $<17.5 ;$ women $\geq 13.8$ and $<15.3$ ) & $743(14.5)$ & $17(7.6)$ & & 0.467 & $36(8.0)$ & $17(7.6)$ & & 0.009 \\
\hline High (men $\geq 17.5 ;$ women $\geq 15.3$ ) & $55(1.1)$ & $3(1.3)$ & & 0.012 & $6(1.3)$ & $3(1.3)$ & & 0.000 \\
\hline Unknown & $1,444(28.2)$ & $10(4.5)$ & & 2.868 & $22(4.9)$ & $10(4.5)$ & & 0.009 \\
\hline Hematocrit, \% & & & & & & & & \\
\hline Low $($ men<41.5; women<35.9) & $1,200(23.4)$ & $132(58.9)$ & & 1.188 & $254(56.7)$ & $132(58.9)$ & & 0.047 \\
\hline Normal low (men $\geq 41.5$ and $<45.9$; women $\geq 35.9$ and $<40.2$ ) & $1,558(30.4)$ & $55(24.6)$ & & 0.322 & $121(27)$ & $55(24.6)$ & & 0.053 \\
\hline Normal high (men $\geq 45.9$ and $<50.4$; women $\geq 40.2$ and $<44.6$ ) & $818(16.0)$ & $21(9.4)$ & $<0.001$ & 0.421 & $39(8.7)$ & $21(9.4)$ & 0.950 & 0.014 \\
\hline High $($ men $\geq 50.4$; women $\geq 44.6$ ) & $99(1.9)$ & $6(2.7)$ & & 0.032 & $11(2.5)$ & $6(2.7)$ & & 0.005 \\
\hline Unknown & $1,450(28.3)$ & $10(4.5)$ & & 2.888 & $23(5.1)$ & $10(4.5)$ & & 0.014 \\
\hline Lymphocyte, \% & & & & & & & & \\
\hline Low $(<20)$ & $780(15.2)$ & $88(39.3)$ & & 0.797 & $173(38.6)$ & $88(39.3)$ & & 0.014 \\
\hline Normal low ( $\geq 20$ and $<32$ ) & $1,482(28.9)$ & $63(28.1)$ & & 0.040 & $142(31.7)$ & $63(28.1)$ & & 0.073 \\
\hline Normal high ( $\geq 32$ and $<44$ ) & $1,102(21.5)$ & $45(20.1)$ & $<0.001$ & 0.073 & $75(16.7)$ & $45(20.1)$ & 0.651 & 0.069 \\
\hline High $(\geq 44)$ & $294(5.7)$ & $17(7.6)$ & & 0.081 & $29(6.5)$ & $17(7.6)$ & & 0.023 \\
\hline Unknown & $1,467(28.6)$ & $11(4.9)$ & & 2.758 & $29(6.5)$ & $11(4.9)$ & & 0.035 \\
\hline Platelets, $\mu \mathrm{L}$ & & & & & & & & \\
\hline Low $(<150 K)$ & $434(8.5)$ & $52(23.2)$ & & 0.477 & $90(20.1)$ & $52(23.2)$ & & 0.065 \\
\hline Normal low ( $\geq 150 \mathrm{~K}$ and $<300 \mathrm{~K})$ & $2,541(49.6)$ & $137(61.2)$ & & 0.526 & $273(60.9)$ & $137(61.2)$ & & 0.005 \\
\hline Normal high ( $\geq 300 \mathrm{~K}$ and $<450 \mathrm{~K})$ & $646(12.6)$ & $23(10.3)$ & $<0.001$ & 0.128 & $57(12.7)$ & $23(10.3)$ & 0.799 & 0.054 \\
\hline High $(\geq 450 \mathrm{~K})$ & $61(1.2)$ & $2(0.9)$ & & 0.017 & $5(1.1)$ & $2(0.9)$ & & 0.005 \\
\hline Unknown & $1,443(28.2)$ & $10(4.5)$ & & 2.865 & $23(5.1)$ & $10(4.5)$ & & 0.014 \\
\hline
\end{tabular}


Table 2 (continued)

\begin{tabular}{|c|c|c|c|c|c|c|c|c|}
\hline \multirow[t]{2}{*}{ Characteristics } & \multicolumn{4}{|l|}{ Before PSM } & \multicolumn{4}{|l|}{ After PSM } \\
\hline & $\begin{array}{l}\text { control } \\
(N=5,125)\end{array}$ & $\begin{array}{l}\text { dementia } \\
(N=224)\end{array}$ & $p$ value & SMD & $\begin{array}{l}\text { control } \\
(N=448)\end{array}$ & $\begin{array}{l}\text { dementia } \\
(N=224)\end{array}$ & $p$ value & SMD \\
\hline \multicolumn{9}{|l|}{ White blood cell, $\mu \mathrm{L}$} \\
\hline $\operatorname{Low}(<4,500)$ & $948(18.5)$ & $69(30.8)$ & & 0.489 & $119(26.6)$ & $69(30.8)$ & & 0.088 \\
\hline Normal $(\geq 4,500$ and $<11,000)$ & $2,593(50.6)$ & $134(59.8)$ & $<0.001$ & 0.427 & $281(62.7)$ & $134(59.8)$ & 0.692 & 0.062 \\
\hline $\operatorname{High}(\geq 11,000)$ & $141(2.8)$ & $11(4.9)$ & & 0.083 & $24(5.4)$ & $11(4.9)$ & & 0.009 \\
\hline Unknown & $1,443(28.2)$ & $10(4.5)$ & & 2.865 & $24(5.4)$ & $10(4.5)$ & & 0.019 \\
\hline \multicolumn{9}{|l|}{ Outcomes, $n(\%)$} \\
\hline \multicolumn{9}{|c|}{ Using the intensive care unit during hospitalization } \\
\hline No/unknown & $4,954(96.7)$ & $208(92.9)$ & 0.004 & & $404(90.2)$ & $208(92.9)$ & 0.315 & \\
\hline Yes & $171(3.3)$ & $16(7.1)$ & & & $44(9.8)$ & $16(7.1)$ & & \\
\hline \multicolumn{9}{|l|}{ Death, $N$} \\
\hline No & $4,966(96.9)$ & $149(66.5)$ & $<0.001$ & & $376(83.9)$ & $149(66.5)$ & $<0.001$ & \\
\hline Yes & $159(3.1)$ & $75(33.5)$ & & & $72(16.1)$ & $75(33.5)$ & & \\
\hline \multicolumn{9}{|l|}{ Death within 30 days } \\
\hline No & $4,995(97.5)$ & $157(70.1)$ & $<0.001$ & & $385(85.9)$ & $157(70.1)$ & $<0.001$ & \\
\hline Yes & $130(2.5)$ & $67(29.9)$ & & & $63(14.1)$ & $67(29.9)$ & & \\
\hline \multicolumn{9}{|l|}{ Death within 14 days } \\
\hline No & $5,037(98.3)$ & $171(76.3)$ & $<0.001$ & & $407(90.8)$ & $171(76.3)$ & $<0.001$ & \\
\hline Yes & $88(1.7)$ & $53(23.7)$ & & & $41(9.2)$ & $53(23.7)$ & & \\
\hline
\end{tabular}

SMD, standardized mean difference; NA, not applicable; PSM, propensity score matching; COVID-19, coronavirus disease 2019; BMI, body mass index.

Among the dementia patients, 53 (23.7\%) died within 14 days and 67 (29.9\%) within 30 days after COVID-19 diagnosis. That is, $70.7 \%(53 / 75)$ of the deaths occurred within 14 days in dementia. Of the 5,125 control patients, 159 (3.1\%) died. Among them, 88 patients (1.7\%) died within 14 days, and 130 (2.5\%) died within 30 days after COVID-19 diagnosis. Among the controls, 55.3\% (88/159) of the deaths occurred within 14 days. Of the 141 deaths within 14 days after COVID-19 diagnosis, 53 (37.6\%) patients had dementia.

To analyze the effect of dementia on early death after COVID-19 diagnosis more accurately by minimizing effects of other variables, PSM between dementia patients and controls was performed (Table 2). The PSM showed that patients over 70 years old were corrected from $13.1 \%$ to $85.7 \%$ in the control and dementia groups before correction and to $82.8 \%$ and $85.7 \%$ after correction. There were no significant differences between the control and dementia groups in all variables after PSM (all $p$ values $>0.05$; all standardized mean differences $<0.25$ ). The Kaplan-Meier survival estimate between the control and dementia patients showed a downward slope of the survival curve, showing that death within 14 days in dementia patients was more pronounced than the control (Fig. 1). Even after PSM, survival curve showed that patients with dementia had a significantly lower survival rate than the control $(p<0.001)$. The mortality rate within 14 days after COVID-19 diagnosis of the dementia patients was sig- nificantly higher than that of controls before PSM $(p<$ $0.001)$ and even after PSM $(23.7 \%$ vs. $9.2 \%, p<0.001)$ (Table 2).

After adjustment of all covariates in dementia-based matched cohort, the HRs of dementia for death within 30 days and 14 days after COVID-19 infection were 3.757 (95\% CI, 2.281-4.255, $p<0.001)$ and 5.104 (95\% CI, $2.889-5.673, p<0.001$ ), respectively. These high and significant HRs suggest that dementia itself is an independent risk factor for early death after COVID-19 infection.

\section{Discussion}

Our study showed dementia as a major risk factor for early death after COVID-19 infection diagnosis. Our study provides insight that comparable with emergency symptoms, such as dyspnea and loss of conscious, dementia is an independent risk factor for early death, indicating the need for intensive monitoring and urgent and active intervention for dementia the point of COVID-19 diagnosis.

Dementia patients were older and underweight, and had a higher rate of underlying disease than the control group. In addition, the population rates of lower-thannormal levels of hemoglobin, lymphocyte, and platelets were higher than the control group, and these were risk factors for early death after COVID-19 infection. In com- 
Table 3. General logistic regression of death within 30 days after COVID-19 diagnosis

\begin{tabular}{|c|c|c|c|}
\hline Characteristics & $\mathrm{HR}$ & $95 \% \mathrm{Cl}$ & $p$ value \\
\hline \multicolumn{4}{|l|}{ Age, years } \\
\hline$<50$ & Reference & & \\
\hline$\geq 50$ and $<70$ & 7.797 & $(2.128-9.096)$ & 0.002 \\
\hline$\geq 70$ & 34.497 & (9.480-35.789) & $<0.001$ \\
\hline Sex - female & 0.632 & $(0.419-1.045)$ & 0.030 \\
\hline \multicolumn{4}{|l|}{ BMI } \\
\hline $\operatorname{Low}(<18.5)$ & 3.212 & $(1.389-4.051)$ & 0.006 \\
\hline Normal low $(\geq 18.5$ and $<23.0)$ & Reference & & \\
\hline Unknown & 4.045 & $(2.384-4.574)$ & $<0.001$ \\
\hline \multicolumn{4}{|l|}{ Temperature, ${ }^{\circ} \mathrm{C}$} \\
\hline $\operatorname{Low}(<36.0)$ & 6.423 & $(1.396-7.949)$ & 0.017 \\
\hline Normal low $(\geq 36.0$ and $<37.0)$ & Reference & & \\
\hline Normal high $(\geq 37.0$ and $<38.0)$ & 1.710 & $(1.098-2.154)$ & 0.018 \\
\hline $\operatorname{High}(\geq 38.0)$ & 2.421 & $(1.275-3.062)$ & 0.007 \\
\hline Unknown & 6.958 & $(1.073-8.827)$ & 0.042 \\
\hline Sore throat & 0.360 & $(0.137-1.326)$ & 0.038 \\
\hline Shortness of breath & 2.658 & $(1.724-3.091)$ & $<0.001$ \\
\hline Headache & 0.276 & $(0.111-1.188)$ & 0.006 \\
\hline Change of consciousness & 8.395 & $(2.795-9.495)$ & $<0.001$ \\
\hline Diabetes mellitus & 1.906 & $(1.264-2.317)$ & 0.002 \\
\hline Cancer & 3.109 & $(1.440-3.878)$ & 0.004 \\
\hline Dementia & 4.543 & $(2.788-5.031)$ & $<0.001$ \\
\hline \multicolumn{4}{|l|}{ Hemoglobin, g/dL } \\
\hline Lower (men <12.3; women <10.8) & 1.917 & $(1.134-2.441)$ & 0.015 \\
\hline Normal low (men $\geq 14.0$ and $<15.7$; women $\geq 12.3$ and $<13.8$ ) & Reference & & \\
\hline \multicolumn{4}{|l|}{ Lymphocyte, \% } \\
\hline Low $(<20)$ & 3.812 & $(2.233-4.347)$ & $<0.001$ \\
\hline Normal low ( $\geq 20$ and $<32$ ) & Reference & & \\
\hline Unknown & 5.265 & $(1.339-6.634)$ & 0.017 \\
\hline \multicolumn{4}{|l|}{ Platelets, $\mu \mathrm{L}$} \\
\hline Low $(<150 K)$ & 2.537 & $(1.566-3.019)$ & $<0.001$ \\
\hline Normal low ( $\geq 150 \mathrm{~K}$ and $<300 \mathrm{~K})$ & Reference & & \\
\hline \multicolumn{4}{|l|}{ White blood cell, $\mu \mathrm{L}$} \\
\hline Normal $(\geq 4,500$ and $<11,000)$ & Reference & & \\
\hline High $(\geq 11,000)$ & 3.595 & $(1.970-4.196)$ & $<0.001$ \\
\hline
\end{tabular}

$\mathrm{HR}$, hazard ratio; $\mathrm{Cl}$, confidence interval; COVID-19, coronavirus disease 2019; BMI, body mass index.

parison, the number of common symptom complaints caused by COVID-19 was lower than that of the control group, which is thought to decrease the early diagnosis rate of COVID-19 in dementia patients, which may cause an increase in mortality. The reason for the low number of complaints may be due to the decreased communication ability of dementia patients, but may also be a specific biological characteristic of dementia patients. Therefore, in dementia patients, even if the complaints are not serious, it is advisable to conduct a thorough diagnostic test when exposed to risk factors. However, even after PSM of all differences with the control group, dementia patients still showed a higher early mortality rate than the control group, suggesting that another pathological cause of dementia increases the mortality rate.

The APOE $\varepsilon 4 / \varepsilon 4$ genotype increases the risk of Alzheimer's disease by amyloid $\beta$-peptide deposition in senile plagues and cerebral vessels [9]. In an analysis using the UK Biobank, APOE $\varepsilon 4 / \varepsilon 4$ homozygotes have 2.2 -fold higher risk for COVID-19 positivity and 4.3 -fold increase in mortality after COVID-19 than in $\varepsilon 3 / \varepsilon 3$ homozygotes [8]. Apoprotein E4 (APOE4) isoform links to various viral infections $[14,15]$. It facilitates entry of herpes simplex virus type-1 particles into the cell and promotes the viral colonization in the brain with higher efficiency than APOE3 [14]. Also, APOE4 increased the rate of human 
Table 4. General logistic regression of death within 14 days after COVID-19 diagnosis

\begin{tabular}{|c|c|c|c|}
\hline Characteristics & $\mathrm{HR}$ & $95 \% \mathrm{Cl}$ & $p$ value \\
\hline \multicolumn{4}{|l|}{ Age (years) } \\
\hline$<50$ & Reference & & \\
\hline$\geq 50$ and $<70$ & 14.533 & $(1.882-16.577)$ & 0.010 \\
\hline$\geq 70$ & 53.959 & $(6.995-56.002)$ & $<0.001$ \\
\hline \multicolumn{4}{|l|}{$\mathrm{BMI}$} \\
\hline Normal low $(\geq 18.5$ and $<23.0)$ & Reference & & \\
\hline Unknown & 3.246 & $(1.820-3.825)$ & $<0.001$ \\
\hline \multicolumn{4}{|l|}{ Temperature, ${ }^{\circ} \mathrm{C}$} \\
\hline Low $(<36.0)$ & 7.319 & $(1.490-8.911)$ & 0.014 \\
\hline Normal low $(\geq 36.0 \&<37.0)$ & Reference & & \\
\hline Normal high $(\geq 37.0$ and $<38.0$ ) & 2.014 & $(1.230-2.507)$ & 0.005 \\
\hline High $(\geq 38.0)$ & 3.575 & $(1.810-4.255)$ & $<0.001$ \\
\hline Unknown & 17.292 & $(4.658-18.604)$ & $<0.001$ \\
\hline Shortness of breath & 2.478 & $(1.549-2.947)$ & $<0.001$ \\
\hline Headache & 0.299 & $(0.107-1.324)$ & 0.021 \\
\hline Change of consciousness & 9.858 & (3.494-10.896) & $<0.001$ \\
\hline Diabetes mellitus & 2.210 & $(1.416-2.654)$ & $<0.001$ \\
\hline Dementia & 5.175 & $(3.073-5.696)$ & $<0.001$ \\
\hline \multicolumn{4}{|l|}{ Hemoglobin, $\mathrm{g} / \mathrm{dL}$} \\
\hline Lower (men <12.3; women <10.8) & 2.248 & $(1.270-2.819)$ & 0.005 \\
\hline Normal low (men $\geq 14.0$ and $<15.7$; women $\geq 12.3$ and $<13.8$ ) & Reference & & \\
\hline Unknown & 33.019 & $(1.757-35.952)$ & 0.019 \\
\hline \multicolumn{4}{|l|}{ Lymphocyte, \% } \\
\hline Low $(<20)$ & 3.492 & $(1.871-4.116)$ & $<0.001$ \\
\hline Normal low ( $\geq 20$ and $<32$ ) & Reference & & \\
\hline Unknown & 6.585 & $(1.590-8.006)$ & 0.009 \\
\hline \multicolumn{4}{|l|}{ Platelets, $\mu \mathrm{L}$} \\
\hline Low $(<150 K)$ & 2.411 & $(1.417-2.942)$ & 0.001 \\
\hline Normal low ( $\geq 150 \mathrm{~K}$ and $<300 \mathrm{~K})$ & Reference & & \\
\hline \multicolumn{4}{|l|}{ White blood cell, $\mu \mathrm{L}$} \\
\hline Normal $(\geq 4,500$ and $<11,000)$ & Reference & & \\
\hline High $(\geq 11,000)$ & 3.571 & $(1.881-4.212)$ & $<0.001$ \\
\hline
\end{tabular}

$\mathrm{HR}$, hazard ratio; $\mathrm{Cl}$, confidence interval; COVID-19, coronavirus disease 2019; BMI, body mass index.

immunodeficiency virus cell entry and disease progression [15]. One study speculated that coronavirus might bind to the APOE protein and APOE cluster genes and neighboring genes, which mediate inflammation and may interact with COVID-19, resulting in high susceptibility and poor prognosis in dementia patients [16]. APOE may relate to angiotensin-converting enzyme 2 (ACE2), an entry receptor of severe acute respiratory syndrome coronavirus 2. In mouse Alzheimer's disease model, the ACE2 gene highly expresses in brain [17]. The majority of the ACE2-expressing cells in the lung are type II alveolar cells, and APOE gene is one of the highly expressed viral process-related genes in ACE2-expressing type II alveolar cells [18].

Increased inflammatory response after COVID-19 infection might have resulted in the early death in dementia patients due to various causes. The APOE4 affects pro-/ anti-inflammatory actions by moderating macrophage [19]. A previous study showed that viral exacerbations of asthma and increased APOE concentrations from bronchoalveolar lavage fluid macrophages amplify pulmonary inflammatory responses [20]. Inflammatory cytokine plasma level, including interleukin-6 (IL-6), is higher in dementia patients than their age-matched controls. The serum IL-6 level positively correlated with dementia progression and inversely related to the immune response in other studies [21,22]. An elevated serum IL-6 level and increased fatality were observed in critically ill COVID-19 patients [23]. Elevated IL-6 is central to the development of macrophage activation syndrome cytokine storm [24]. Cytokine storm causes acute respiratory distress syndrome and multi-organ failure, leading to death [25]. 


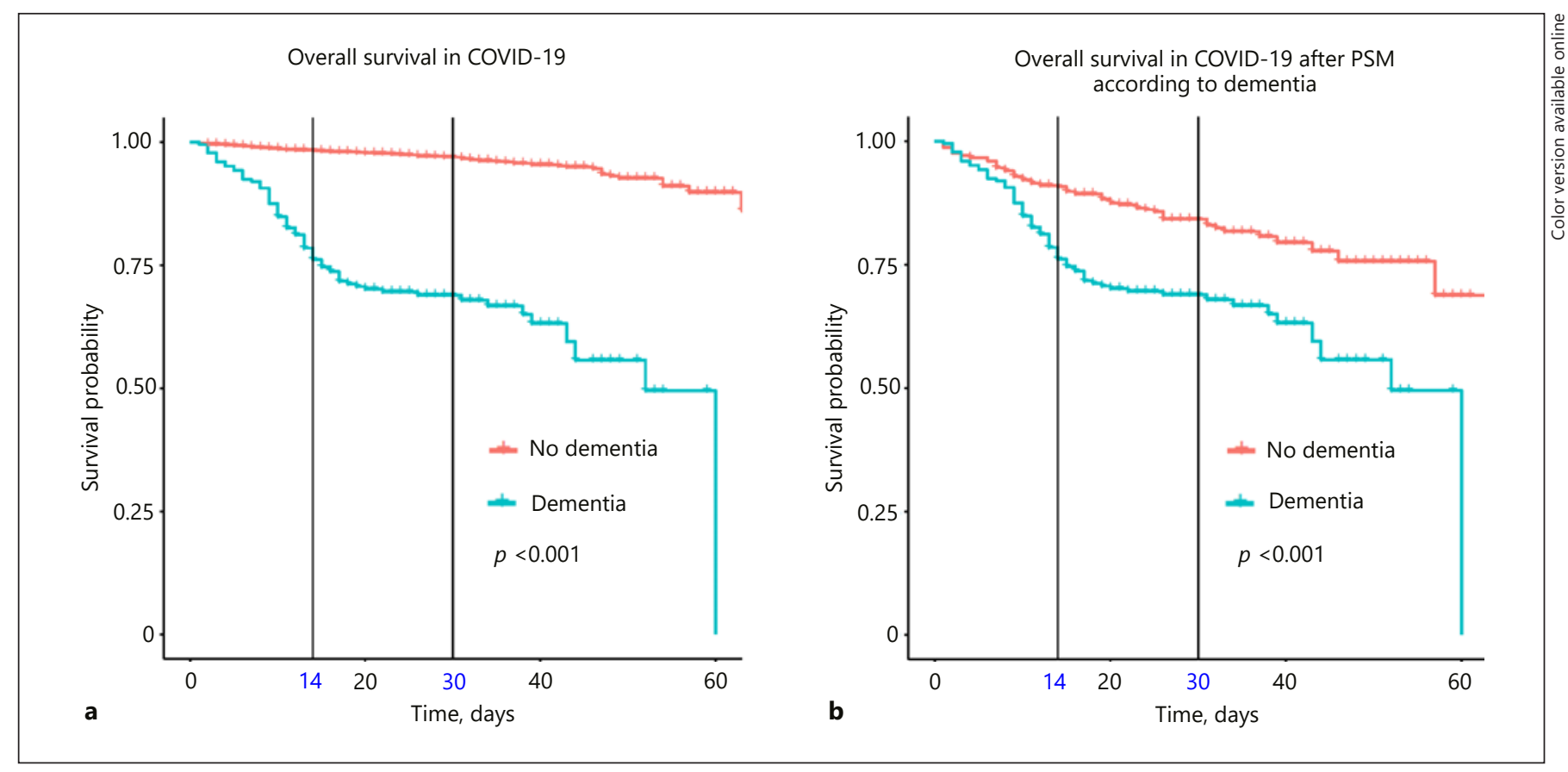

Fig. 1. Kaplan-Meier overall survival estimate according to the dementia comorbidity after COVID-19 diagnosis. a Before PSM. b After PSM $p$ value, log-rank test. PSM, propensity score matching; COVID-19, coronavirus disease 2019.

The premature early death of dementia patients might be influenced by other socio-clinical factors; for example, the loss of communication ability can delay COVID-19 diagnosis and underestimate the disease severity [11]. In addition, it is difficult to prepare medical and human resources for COVID-19 dementia patients. Particularly, a resident COVID-19-negative caregiver who will accept quarantine conditions is needed for dementia patients [26]. Finally, cohort quarantine policies for nursing hospitals with confirmed COVID-19 infections may hinder timely intervention in patients with dementia [27]. All these COVID-19 quarantine policies and conditions can cause undue difficulty for dementia patients, leading to early mortality within 14 days of COVID-19 diagnosis. To prevent premature dementia patients' death after COVID-19 infection, close monitoring, prompt isolation, and active treatments are necessary.

Among the underlying diseases, diabetes, as well as dementia, was analyzed as a major risk factor for early death after COVID-19 infection. Several studies have demonstrated heterogeneous results regarding the impact of diabetes medications on the prognosis of COVID-19 patients. Metformin reduced mortality from COVID-19 infection [28], whereas dipeptidyl peptidase 4 inhibitors did not affect the outcome of COVID-19 [29]. KDCA did not provide information on the patient's diabetes medications. Further research is essential to evaluate the effect of diabetes as an underlying disease and each diabetes drug on early death in COVID-19.

Our study also showed that low hemoglobin, lymphocytes, and platelets were significantly correlated with early death from COVID-19. Anemia [30], lymphopenia [31], and thrombocytopenia [32] were reported as risk factors for severe COVID-19 infection, respectively. In the case of hemoglobin, a study insisted that COVID-19 should be regarded as an oxygen-deprived blood disease and improvement of hemoglobin dysfunction could be a therapeutic approach for this disease.

This study has limitations as a retrospective study. For example, patients with missing values (category of "unknown") on BMI, body temperature, and blood tests showed a much worse prognosis than the reference groups with normal range values, suggesting that information input may have been omitted in patients who needed emergency treatment due to severe symptoms at the time of COVID-19 diagnosis. As the COVID-19 pandemic prolongs, a systematic prospective study will help to define risk factors of a poor prognosis more accurately in COVID-19. 


\section{Conclusion}

Dementia patients are at higher risk of early death within 14 days of COVID-19 diagnosis. It is necessary to prevent COVID-19 in dementia patients thoroughly, and intensive monitoring is necessary to prevent early death of dementia patients after COVID-19 diagnosis. Further randomized controlled studies are needed to confirm the finding of this study that dementia is an independent risk factor for early death from COVID-19.

\section{Statement of Ethics}

Study approval statement: This study protocol was reviewed and approved by the Institutional Review Board of Ewha Womans University Medical Center, approval number [SEUMC 2020-09009]. Consent to participate statement: This study has been granted an exemption from requiring written informed consent by the Institutional Review Board of Ewha Womans University Medical Center.

\section{Conflict of Interest Statement}

The authors declare that they have no competing interests.

\section{Funding Sources}

This work was supported by the National Research Foundation of Korea (NRF, https://www.nrf.re.kr/eng/index) grant funded by the Ministry of Science and ICT (No. 2020R1C1C1011369) and a grant of the Korea Health Technology R\&D Project through the Korea Health Industry Development Institute (KHIDI), funded by the Ministry of Health \& Welfare, Republic of Korea (No. HI21C1218) to Y.J.Kim,2019M3C1B8090802,2019M3C1B8090803 and 2020R1A2C2013216 to H.W. Lee. The funders had no role in study design, data collection and analysis, decision to publish, or preparation of the manuscript.

\section{Author Contributions}

Y.J. Kim planned the study, performed all statistical analyses, supervised the data analysis, and wrote the paper. Y. Jee performed data acquisition and contributed to revising the paper. E. H. Ha, I. Jo, H.W. Lee, and M.S. Song helped to plan the study and to revise the manuscript.

\section{Data Availability Statement}

The data that support the findings of this study are available from the KDCA, but restrictions apply to the availability of these data, which were used under license for the current study, and so are not publicly available. Data are however available from the authors upon reasonable request and with permission of KDCA.

\section{References}

1 WHO. WHO coronavirus disease (COVID-19) dashboard. Accessed from 2021 Jan 12.

2 Puls A, Bracke S. COVID-19 pandemic risk analytics: data mining with reliability engineering methods for analyzing spreading behavior and comparison with infectious diseases. medRxiv. 2020.

3 Mahase E. Covid-19: Pfizer vaccine efficacy was $52 \%$ after first dose and $95 \%$ after second dose, paper shows. BMJ. 2020;371:m4826.

4 Tanne JH. Covid-19: FDA panel votes to approve Pfizer BioNTech vaccine. BMJ. 2020; 371:m4799.

5 McDonnell A, Van Exan R, Lloyd S, Subramanian L, Chalkidou K, La Porta A, et al. COVID-19 vaccine predictions: using mathematical modelling and expert opinions to estimate timelines and probabilities of success of COVID-19 vaccines. Washington, DC: Center for Global Development; 2020.

6 Zheng Z, Peng F, Xu B, Zhao J, Liu H, Peng J, et al. Risk factors of critical \& mortal COVID-19 cases: a systematic literature review and meta-analysis. J Infect. 2020;81(2):e1625.
7 Zhou F, Yu T, Du R, Fan G, Liu Y, Liu Z, et al. Clinical course and risk factors for mortality of adult inpatients with COVID-19 in Wuhan, China: a retrospective cohort study. Lancet. 2020;395(10229):1038.

8 Kuo CL, Pilling LC, Atkins JL, Masoli JAH, Delgado J, Kuchel GA, et al. ApoE e4e4 genotype and mortality with COVID-19 in UK Biobank. J Gerontol A Biol Sci Med Sci. 2020; 75(9):1801-3

9 Schmechel DE, Saunders AM, Strittmatter WJ, Crain BJ, Hulette CM, Joo SH, et al. Increased amyloid beta-peptide deposition in cerebral cortex as a consequence of apolipoprotein E genotype in late-onset Alzheimer disease. Proc Natl Acad Sci U S A. 1993; 90(20):9649-53.

10 Sadigh-Eteghad S, Talebi M, Farhoudi M. Association of apolipoprotein E epsilon 4 allele with sporadic late onset Alzheimer's disease. A meta-analysis. Neurosciences. 2012 Oct; 17(4):321-6.

11 Bianchetti A, Rozzini R, Guerini F, Boffelli S, Ranieri P, Minelli G, et al. Clinical presentation of COVID19 in dementia patients. J Nutr Health Aging. 2020;24:560.
12 Hariyanto TI, Putri C, Situmeang RFV, Kurniawan A. Dementia is a predictor for mortality outcome from coronavirus disease 2019 (COVID-19) infection. Eur Arch Psychiatry Clin Neurosci. 2021:271(2):393-5.

13 McPherson RA, Msc M, Pincus MR. Henry's clinical diagnosis and management by laboratory methods E-book. Elsevier Health Sciences; 2021.

14 Dobson CB, Sales SD, Hoggard P, Wozniak $\mathrm{MA}$, Crutcher KA. The receptor-binding region of human apolipoprotein $\mathrm{E}$ has direct anti-infective activity. J Infect Dis. 2006; 193(3):442-50.

15 Burt TD, Agan BK, Marconi VC, He W, Kulkarni H, Mold JE, et al. Apolipoprotein (apo) E4 enhances HIV-1 cell entry in vitro, and the APOE $\varepsilon 4 / \varepsilon 4$ genotype accelerates HIV disease progression. Proc Natl Acad Sci U S A. 2008;105(25):8718-23.

16 Finch CE, Kulminski AM. The ApoE locus and COVID-19: are we going where we have been? J Gerontol A Biol Sci Med Sci. 2020; 76(2):e1-3.

17 Lim KH, Yang S, Kim SH, Joo JY. Elevation of ACE2 as a SARS-CoV-2 entry receptor gene expression in Alzheimer's disease. J Infect. 2020 Sep;81(3):e33-4. 
18 Zhao Y, Zhao Z, Wang Y, Zhou Y, Ma Y, Zuo W. Single-cell RNA expression profiling of ACE2, the putative receptor of Wuhan 2019nCov. BioRxiv. 2020.

19 Tudorache IF, Trusca VG, Gafencu AV. Apolipoprotein E: a multifunctional protein with implications in various pathologies as a result of its structural features. Comput Struct Biotechnol J. 2017;15:359-65.

20 Gordon EM, Yao X, Xu H, Karkowsky W, Kaler M, Kalchiem-Dekel O, et al. Apolipoprotein $\mathrm{E}$ is a concentration-dependent pulmonary danger signal that activates the NLRP 3 inflammasome and IL- $1 \beta$ secretion by bronchoalveolar fluid macrophages from asthmatic subjects. J Allergy Clin Immunol. 2019;144(2):426-e3.

21 Motta M, Imbesi R, Di Rosa M, Stivala F, Malaguarnera L. Altered plasma cytokine levels in Alzheimer's disease: correlation with the disease progression. Immunol Lett. 2007; 114(1):46-51.

22 Cojocaru IM, Cojocaru M, Miu G, Sapira V. Study of interleukin-6 production in Alzheimer's disease. Rom J Intern Med. 2011; 49(1):55-8.
23 Chen X, Zhao B, Qu Y, Chen Y, Xiong J, Feng $\mathrm{Y}$, et al. Detectable serum severe acute respiratory syndrome coronavirus 2 viral load (RNAemia) is closely correlated with drastically elevated interleukin 6 level in critically ill patients with coronavirus disease 2019. Clin Infect Dis. 2020 Nov 5;71(8):1937-42.

24 McGonagle D, Sharif K, O'Regan A, Bridgewood C. The role of cytokines including interleukin-6 in COVID-19 induced pneumonia and macrophage activation syndrome-like disease. Autoimmun Rev. 2020 Jun;19(6):102537.

25 Mauvais-Jarvis F. Aging, male sex, obesity, and metabolic inflammation create the perfect storm for COVID-19. Diabetes. 2020 Sep; 69(9):1857-63.

26 WHO. Additional COVID-19 guidance for caregivers of people living with dementia in community settings. Centers for Disease Control and Prevention; 2020.

27 Nursing home residents in S. Korea are being 'quarantined to death', The Korea Herald/Asia News Network. 2021;03:02 PM 2021 Jan 6.
28 Hariyanto TI, Kurniawan A. Metformin use is associated with reduced mortality rate from coronavirus disease 2019 (COVID-19) infection. Obes Med. 2020 Sep;19:100290.

29 Hariyanto TI, Kurniawan A. Dipeptidyl peptidase 4 (DPP4) inhibitor and outcome from coronavirus disease 2019 (COVID-19) in diabetic patients: a systematic review, meta-analysis, and meta-regression. J Diabetes Metab Disord. 2021;20(1):1-8.

30 Hariyanto TI, Kurniawan A. Anemia is associated with severe coronavirus disease 2019 (COVID-19) infection. Transfus Apher Sci. 2020;59(6):102926.

31 Tan L, Wang Q, Zhang D, Ding J, Huang Q, Tang Y-Q, et al. Lymphopenia predicts disease severity of COVID-19: a descriptive and predictive study. Signal Transduct Target Ther. 2020;5(1):1-3.

32 Lippi G, Plebani M, Henry BM. Thrombocytopenia is associated with severe coronavirus disease 2019 (COVID-19) infections: a metaanalysis. Clin Chim Acta. 2020;506:145-8. 